\title{
Solvability and dynamics study of a weak solution for a nonlinear parabolic problem with the bessel operator
}

Research Article

\author{
Oussaeif Taki-Eddine ${ }^{1 * \dagger}$ and Chebana Zainouba ${ }^{2} \ddagger$ \\ 1,2 Department of Mathematics and Informatics, Oum El Bouaghi University, Dynamic and control systems laboratory, \\ Oum El Bouaghi, Algeria
}

\begin{abstract}
In this paper we present a class of parabolic equation with nonlinear where we show two part of this study the theoretical part we prove the existence and uniqueness of the solution by energy inequality method. Then we present finite time blow up by using the energy method.
\end{abstract}

MSC: $\quad 35 \mathrm{~K} 58,35 \mathrm{~K} 61,35 \mathrm{~B} 45,35 \mathrm{~A} 01,35 \mathrm{~A} 02$

Keywords: Parabolic equation• Non linear eqautions• Existence• Uniqueness Energy inequality method

\section{Introduction}

Partial differential equations play an important role in modeling many disciplines including engineering, physics, chemistry economics, and biology, and their study has occupied mathematicians since the eighteenth century with the work of Euler, D'Alambert, Lagrange, and Laplace...; over the last forty years many modern physical, mechanical, biological and technological phenomena, and problems have been shaped by partial differential equations (PDEs), many physical phenomena are modeled by nonclassical parabolic problems associated with boundary conditions.

As the first question to be asked in the theoretical study is to know if, for a nonlinear evolution equation with initial conditions and boundary conditions, there exists at least one local solution and if it is unique in the case considered, these problems have been solved for a large class of nonlinear evolution equations by a series of useful

\footnotetext{
* E-mail: taki_maths@live.fr

$\dagger$ E-mail: takieddine.oussaeif@gmail.com

¥ E-mail: chebanazainouba@yahoo.com
} 
methods and theories which have been developed, in particular since the 1960s, such as the method of Faedo Galerkin (Compactness method), the fixed point method, the semi-group method and the monotonic iterative method (For more details on these methods also the energy inequalities method [1-12]. Hence, it is important to find the solution of the mathematical model in order to resolve the engineering problems.

On the other hand, the dynamic questions in the field of partial differential equations problems like the blowup and decay studies are considered among the most important issues because they fall under the framework of controlling the studied phenomenon. Blow-up singularity, as one of the most remarkable properties that distinguish nonlinear parabolic problems from the linear ones, attracted extensive attention of mathematicians in the past few decades.

There are many works focused on the blow-up property of various parabolic equations (or systems) with homogenous Newmann boundary conditions.

\section{Formulation of the nonlinear problem}

Let $Q=\left\{(x, t) \in \mathbb{R}^{2}, 0<x<1\right.$ and $\left.0<t<T\right\}$.

Consider the following nonlinear problem :

$$
\begin{cases}\frac{\partial u}{\partial t}-a \frac{1}{x} \frac{\partial}{\partial x}\left(x \frac{\partial u}{\partial x}\right)+b u=f\left(x, t, u, \frac{\partial u}{\partial x}\right), & \forall(x, t) \in Q \\ u(x, 0)=\varphi(x), & \forall x \in(0,1) \\ \frac{\partial u}{\partial x}(0, t)=\frac{\partial u}{\partial x}(1, t)=0 . & \forall t \in(0, T)\end{cases}
$$

Such that $a, b>0, f, \varphi \in L^{2}(Q)$ are known functions, with the following condition :

The function $f$ is lipchitzian, i.e : there exists a positive constant $k$ such that :

$$
\begin{gathered}
\left\|f\left(x, t, u_{1}, v_{1}\right)-f\left(x, t, u_{2}, v_{2}\right)\right\|_{L^{2}(Q)} \leq k\left(\left\|u_{1}-u_{2}\right\|_{L^{2}(Q)}+\left\|v_{1}-v_{2}\right\|_{L^{2}(Q)}\right), \\
\forall\left(u_{1}, v_{1}\right),\left(u_{2}, v_{2}\right) \in\left(L^{2}(Q)\right)^{2} .
\end{gathered}
$$

\section{Study of the associated linear problem}

\subsection{Position of the associated linear problem}

In the rectangular area $Q=(0,1) \times(0, T)$, with $T<\infty$, we consider the following linear problem :

$$
\begin{cases}\frac{\partial u}{\partial t}-a \frac{1}{x} \frac{\partial}{\partial x}\left(x \frac{\partial u}{\partial x}\right)+b u=f(x, t) & \forall(x, t) \in Q \\ u(x, 0)=\varphi(x) & \forall x \in(0,1) \\ \frac{\partial u}{\partial x}(0, t)=\frac{\partial u}{\partial x}(1, t)=0 & \forall t \in(0, T)\end{cases}
$$


Whose parabolic equation is given as follows :

$$
\mathcal{L} u=\frac{\partial u}{\partial t}-a \frac{1}{x} \frac{\partial}{\partial x}\left(x \frac{\partial u}{\partial x}\right)+b u=f(x, t)
$$

with the initial condition :

$$
\ell u=u(x, 0)=\varphi(x), \quad x \in(0,1)
$$

the boundry condition of Neuman type :

$$
\frac{\partial u}{\partial x}(0, t)=\frac{\partial u}{\partial x}(1, t)=0
$$

Where $f$ and $\varphi$ are known functions and $a$ and $b$ are a positive constants.

We note that the function $\varphi$ satisfies the compatibility condition (6) :

$$
\frac{\partial \varphi}{\partial x}(1)=0, \frac{\partial \varphi}{\partial x}(0)=0
$$

\subsection{A priori estimate (the uniquenss of the solution)}

The problem (3) can be written in the following operational form :

$$
L u=\mathcal{F}
$$

where $L=(\mathcal{L}, \ell)$, with a domain of definition $D(L)$ consisting of function $u \in L_{\sqrt{x}}^{2}(Q)$, such that $\frac{\partial u}{\partial t}$, $\frac{\partial u}{\partial x}$ and $\frac{\partial^{2} u}{\partial x^{2}} \in L_{\sqrt{x}}^{2}(Q)$ and $u$ satisfies the boundary conditions in (6) ;

The operator $L$ is considered from $E$ to $F$, where $E$ is the Banach space of functions $u \in L_{\sqrt{x}}^{2}(Q)$ and whose norm :

$$
\|u\|_{E}^{2}=\|u\|_{C\left(0, T, L_{\sqrt{x}}^{2}(0,1)\right)}^{2}+\left\|\partial_{x} u\right\|_{L^{2}\left(0, T, L_{\sqrt{x}}^{2}(0,1)\right)}^{2}+\|u\|_{L^{2}\left(0, T, L_{\sqrt{x}}^{2}(0,1)\right)}^{2}
$$

is finite, and $F$ is the Hilbert space of all the elements $\mathcal{F}=(f, \varphi)$ whose norm :

$$
\|\mathcal{F}\|_{F}^{2}=\|f\|_{L^{2}(Q)}^{2}+\|\varphi\|_{L_{\sqrt{x}}^{2}(0,1)}^{2}
$$

is finite.

Theorem 3.1.

For any function $u \in D(L)$, we have the estimate :

$$
\|u\|_{E} \leq k\|L u\|_{F}
$$

where $k$ is a positive constant independent of $u$, such that :

$$
k=\sqrt{\frac{e^{T}}{\min _{\{1,2 a, 2 b\}}}}
$$


Proof. Multiplying the equation of the problem (3) by the following multiplicator $M u$ :

$$
M u=x u(x, t)
$$

and by integrating on the $Q_{\tau}=(0,1) \times(0, \tau)$,where $\tau \in[0, T]$,we obtain :

$$
\begin{aligned}
& \int_{Q_{\tau}}\left[\partial_{t} u(x, t)-a \frac{1}{x} \partial_{x}\left(x \partial_{x} u(x, t)\right)+b u(x, t)\right] \cdot M u(x, t) d x d t \\
= & \int_{Q_{\tau}} \partial_{t} u(x, t) \cdot x u(x, t) d x d t-a \int_{Q_{\tau}} \partial_{x}\left(x \partial_{x} u(x, t)\right) \cdot x u(x, t) d x d t \\
& +\int_{Q_{\tau}} b u(x, t) \cdot x u(x, t) \\
= & \int_{Q_{\tau}} f(x, t) \cdot x u(x, t) d x d t .
\end{aligned}
$$

Then, we get

$$
\begin{aligned}
& \int_{0}^{1} x u(x, \tau)^{2} d x+2 a \int_{Q_{\tau}} x\left(\partial_{x} u(x, t)\right)^{2} d x d t+2 b \int_{Q_{\tau}} x u(x, t)^{2} d x d t \\
\leq & \int_{Q}^{1} f(x, t)^{2} d x d t+\int_{0} x \varphi(x)^{2} d x+\int_{Q} x u(x, t)^{2} d x d t
\end{aligned}
$$

Applying Gronwall's lemma, we find :

$$
\begin{aligned}
& \max _{0<t<T} \int_{0}^{1} x u(x, \tau)^{2} d x+\int_{Q} x\left(\partial_{x} u(x, t)\right)^{2} d x d t+\int_{Q} x u(x, t)^{2} d x d t \\
& \leq \frac{e^{T}}{\min _{\{1,2 a, 2 b\}}}\left(\int_{Q} f(x, t)^{2} d x d t+\int_{0}^{1} x \varphi(x)^{2} d x\right)
\end{aligned}
$$

with

$$
C=\frac{e^{T}}{\min _{\{1,2 a, 2 b\}}}
$$

Thus, we write

$$
\|u\|_{C\left(0, T, L_{\sqrt{x}}^{2}(0,1)\right)}^{2}+\left\|\partial_{x} u\right\|_{L^{2}\left(0, T, L_{\sqrt{x}}^{2}(0,1)\right)}^{2}+\|u\|_{L^{2}\left(0, T, L_{\sqrt{x}}^{2}(0,1)\right)}^{2} \leq C\left(\|f\|_{L^{2}(Q)}^{2}+\|\varphi\|_{L_{\sqrt{x}}^{2}(0,1)}^{2}\right)
$$

Which is equivalent to

$$
\|u\|_{E} \leq k\|\mathcal{L}\|_{F}, \text { where } k=\sqrt{C} \text {. }
$$


Corollary 3.1.

If for any function $u \in D(L)$, we have the following estimate :

$$
\|u\|_{E} \leq k\|\mathcal{F}\|_{F},
$$

then the solution of the problem (3) if it exists, it is unique.

Proof. Let $u_{1}$ and $u_{2}$ two solutions of the problem (3):

$$
\left\{\begin{array}{l}
L u_{1}=\mathcal{F} \\
L u_{2}=\mathcal{F}
\end{array} \Longrightarrow L u_{1}-L u_{2}=0\right.
$$

and since $L$ is linear and according to (9)

$$
\left\|u_{1}-u_{2}\right\|_{E}^{2} \leq k\|0\|_{F}^{2}=0
$$

which gives

$$
u_{1}=u_{2}
$$

\subsection{Existence of the solution}

The demonstration of the existence of the solution is based on the proof of the following items :

1. The operator

$$
L: E \longrightarrow F
$$

has a closure.

2. $R(L)$ is dense in $F$ for any $u \in E$ and for any arbitrary $\mathcal{F}=(f, \varphi) \in F$.

\section{Proposition 3.1.}

The operator $L$ of $E$ in $F$ is closable.

Proof. Let $\left\{u_{n}\right\} \in D(L)$ be a sequence such that :

$$
u_{n} \longrightarrow 0 \text { dans } E
$$

and

$$
L u_{n} \longrightarrow(f ; \varphi) \text { in } F
$$


we must prove that

$$
f \equiv 0 \text { and } \varphi \equiv 0
$$

The convergence of $u_{n}$ to 0 in $E$, so we have :

$$
u_{n} \longrightarrow 0 \text { in } D^{\prime}(Q)
$$

From the continuity of the derivation of $D^{\prime}(Q)$ in $D^{\prime}(Q)$. then relation (13) implies :

$$
\mathcal{L} u_{n} \longrightarrow 0 \text { in } D^{\prime}(Q)
$$

Moreover, the convergence of $\mathcal{L} u_{n}$ towards $f$ in $L^{2}(Q)$ gives that :

$$
\mathcal{L} u_{n} \longrightarrow f \text { in } D^{\prime}(Q)
$$

By vertue of the uniqueness of the limit in $D^{\prime}(Q)$, we conclude from (14) and (15) that

$$
f=0 \text {. }
$$

Then,it is generated from (12) that:

$$
\ell u_{n} \longrightarrow \varphi \text { in } L_{\sqrt{x}}^{2}(0,1)
$$

On the other hand :

$$
\begin{aligned}
\left\|u_{n}\right\|_{E}^{2} & =\left\|u_{n}\right\|_{C\left(0, T, L_{\sqrt{x}}^{2}(0,1)\right)}^{2}+\left\|\partial_{x} u_{n}\right\|_{L^{2}\left(0, T, L_{\sqrt{x}}^{2}(0,1)\right)}^{2}+\left\|u_{n}\right\|_{L^{2}\left(0, T, L_{\sqrt{x}}^{2}(0,1)\right)}^{2} \\
\left\|u_{n}\right\|_{E}^{2} & \geq\left\|u_{n}\right\|_{C\left(0, T, L_{\sqrt{x}}^{2}(0,1)\right)}^{2} \\
\left\|u_{n}\right\|_{E}^{2} & \geq\left\|u_{n}(x, 0)\right\|_{L_{\sqrt{x}}^{2}(0,1)}^{2} .
\end{aligned}
$$

By crossing the limit, we find :

$$
\lim _{n \rightarrow+\infty}\left\|u_{n}\right\|_{E}^{2} \geq\|\varphi(x)\|_{L_{\sqrt{x}}^{2}(0,1)}^{2}
$$

since $u_{n} \longrightarrow 0$ in $E$ thus $\left\|u_{n}\right\|_{E}^{2} \longrightarrow 0$ in $E$, we find :

$$
\|\varphi(x)\|_{L_{\sqrt{x}}^{2}(0,1)}^{2} \leq 0
$$

from where $\varphi=0$.

Let $\bar{L}$ be the closure of $L$, and $D(\bar{L})$ the domaine of definition of $\bar{L}$ : 
Definition 3.1.

The solution of the equation

$$
\bar{L} u=\mathcal{F},
$$

is called the generalized strong solution of the problem (3).

The theorem (3.1) is valid for a strong generalized solution, so ; we have the inequality :

$$
\|u\|_{E} \leq m\|\bar{L} u\|_{F} \forall u \in D(\bar{L})
$$

Consequently, this last inequality leads to the following corollaries :

\section{Corollary 3.2.}

()The solution of the problem (3) if it exists, it is unique and depends continuously on $\mathcal{F} \in F$.

Corollary 3.3.

() The set $R(\bar{L})$ of the operator $\bar{L}$ is equal to the closure $\overline{R(L)}$ of $R(L)$.

Theorem 3.2.

For each $\mathcal{F}=(f, \varphi) \in F$, the problem (3) admits a unique strong solution $u=\bar{L}^{-1} \mathcal{F}$.

\section{Proposition 3.2.}

If for $\omega \in L^{2}(Q)$ and for any $u \in D_{0}(L)$, we have

$$
\int_{Q} \mathcal{L} u \cdot \omega d x d t=0
$$

then $\omega$ vanishes almost everywhere in $Q$.

$D_{0}(L)=\{u \in D(L), u(x, 0)=0\}$

Proof. The scalar product of $F$ is defined by:

$$
(L u, W)_{F}=\int_{Q} \mathcal{L} u \cdot \omega d x d t+\int_{0}^{1}(\ell u) \cdot\left(\omega_{0}\right) d x
$$

where $W=\left(\omega, \omega_{0}\right)$.

The equality (17) can be written as follows :

$$
\int_{Q} \frac{\partial u}{\partial t} \cdot \omega d x d t-a \int_{Q} \frac{1}{x} \frac{\partial}{\partial x}\left(x \frac{\partial u}{\partial x}\right) \cdot \omega d x d t+b \int_{Q} u \cdot \omega d x d t=0
$$

which implies that

$$
\int_{Q} \frac{\partial u}{\partial t} \cdot \omega d x d t+b \int_{Q} u \cdot \omega d x d t=a \int_{Q} \frac{1}{x} \frac{\partial}{\partial x}\left(x \frac{\partial u}{\partial x}\right) \cdot \omega d x d t
$$

where $u, \frac{\partial u}{\partial t}$ and $\frac{\partial u}{\partial x} \in L_{\sqrt{x}}^{2}(Q)$, with $u$ satisfies the boundary conditions of (3). 
We put

$$
u(x, t)=\int_{0}^{t} z(x, \tau) d \tau=\Im_{t} z
$$

by replacing (21) in (20) we get

$$
\int_{Q} z \cdot \omega d x d t+b \int_{Q} \omega\left(\Im_{t} z\right) d x d t=a \int_{Q} \frac{1}{x} \frac{\partial}{\partial x}\left(x \frac{\partial \Im_{t} z}{\partial x}\right) \omega d x d t
$$

During the establishment of the function $\omega$, and from this last equality,we give the function $\omega$ in terms of the function $z$ as follows :

$$
\omega=x \Im_{t} z
$$

since $z$ satisfies the same conditions as the function $u$ in (3), then $z, \frac{\partial z}{\partial x} \in L_{\sqrt{x}}^{2}(Q)$, so $\omega \in L^{2}(Q)$.

Now replacing $\omega$ in (22)we obtain :

$$
\int_{Q} x z \Im_{t} z d x d t+b \int_{Q} x\left(\Im_{t} z\right)^{2} d x d t=a \int_{Q} \Im_{t} z \cdot \frac{\partial}{\partial x}\left(x \frac{\partial \Im_{t} z}{\partial x}\right) d x d t
$$

According to an integration by part and using the boundary condition of Neumann, we get :

$$
\left.\int_{0}^{1} \frac{x}{2}\left(\Im_{t} z\right)^{2}\right|_{\tau=0} ^{\tau=T} d x+b \int_{Q} x\left(\Im_{t} z\right)^{2} d x d t=-a \int_{Q} x\left(\frac{\partial \Im_{t} z}{\partial x}\right)^{2} d x d t \leqslant 0
$$

which gives

$$
\int_{Q} x\left(\Im_{t} z\right)^{2} d x d t=0
$$

So, we obtain

$$
\left(\Im_{t} z\right)=0 .
$$

Therefore, it becomes $u=0$ in $Q$, which gives $\omega=0$ in $Q$. This was to be proved.

Let as now return to the proof of the theorem (3.2).

We consider a function $W=\left(\omega, \omega_{0}\right) \in R(L)^{\perp}$ and for any $u \in D(L)$, then $W$ verifies the following equality :

$$
(L u, W)_{F}=\int_{Q} \mathcal{L} u \cdot \omega d x d t+\int_{0}^{1}(\ell u) \cdot\left(\omega_{0}\right) d x=0 .
$$

Then, we must prove that $W=0$. We suppose $u \in D_{0}(L)$ in the last equality, we obtain :

$$
\int_{Q} \mathcal{L} u \cdot \omega d x d t=0, u \in D_{0}(L)
$$

From the proposition (17), we deduce that $\omega=0$. Thus we have :

$$
\int_{0}^{1}(\ell u) \cdot\left(\omega_{0}\right) d x=0, u \in D(L) .
$$


Since the set of value of the operator $\ell$ is dense everywhere in the Hilbert space $F$ with the norm

$$
\left(\int_{0}^{1}\left[(\ell u)^{2}\right]\right)^{\frac{1}{2}}
$$

the equality (23) implies that $\omega_{0}=0$. Therefore $W=0$ implies $\overline{R(L)}=F$. This which completes the proof of the theorem (3.2).

\section{Existence and uniqueness of a weak solution of the nonlinear problem}

This section is devoted to the proof of the existence, the uniqueness and the continuous dependence of the solution compared to the data of the nonlinear problem (1).

We consider the following auxiliary problem with the homogeneous equation :

$$
\left\{\begin{array}{c}
\frac{\partial w}{\partial t}-a \frac{1}{x} \frac{\partial}{\partial x}\left(x \frac{\partial w}{\partial x}\right)+b w=0 \\
w(x, 0)=\varphi(x) \\
\frac{\partial w}{\partial x}(0, t)=\frac{\partial w}{\partial x}(1, t)=0
\end{array}\right.
$$

if $u$ is a solution of the problem (1) and $w$ is a solution of the problem (24), then $y=u-w$ satisfies the following problem

$$
\begin{aligned}
\frac{\partial y}{\partial t}-a \frac{1}{x} \frac{\partial}{\partial x}\left(x \frac{\partial y}{\partial x}\right)+b y=G\left(x, t, y, \frac{\partial y}{\partial x}\right) & \\
y(x, 0) & =0 \\
\frac{\partial y}{\partial x}(0, t) & =\frac{\partial y}{\partial x}(1, t)=0
\end{aligned}
$$

where $G\left(x, t, y, \frac{\partial y}{\partial x}\right)=f\left(x, t, y+w, \frac{\partial y}{\partial x}+\frac{\partial w}{\partial x}\right)$. As the function $f$ is lipchitzian the $G$ function is also lipchitzian,i.e there exists a positive constant $k$ such that

$$
\begin{aligned}
\left\|G\left(x, t, u_{1}, v_{1}\right)-G\left(x, t, u_{2}, v_{2}\right)\right\|_{L^{2}(Q)} & \leq k\left(\left\|u_{1}-u_{2}\right\|_{L_{\sqrt{x}}^{2}(Q)}-\left\|v_{1}-v_{2}\right\|_{L_{\sqrt{x}}^{2}(Q)}\right) \\
\forall\left(u_{1}, v_{1}\right),\left(u_{2}, v_{2}\right) & \in\left(L^{2}(Q)\right)^{2} .
\end{aligned}
$$

From the result of the previous section, we deduce that the problem (24) has a unique solution which depends continuously on the data. So it remains to prove that the problem (25) - (27) admits a unique weak solution.

First, we propose the concept of studied solution.

Let $v=v(x, t)$ any function of $L^{2}\left(0, T ; H_{\sqrt{x}}^{1}(0,1)\right)$. 
Then, multiplying (25) by $x v$ then we integrate it on $Q$

$$
\begin{aligned}
& \int_{Q} x \frac{\partial y}{\partial t} v d x d t-a \int_{Q} \frac{\partial}{\partial x}\left(x \frac{\partial y}{\partial x}\right) v d x d t+b \int_{Q} x v y d x d t \\
= & \int_{Q} x v G\left(x, t, y, \frac{\partial y}{\partial x}\right) d x d t
\end{aligned}
$$

Next, using integration by part and the conditions on $y$, it comes

$$
\begin{aligned}
& \int_{Q} x \frac{\partial y}{\partial t} v d x d t+a \int_{Q} x \frac{\partial y}{\partial x} \frac{\partial v}{\partial x} d x d t+b \int_{Q} x v y d x d t \\
= & \int_{Q} x v G\left(x, t, y, \frac{\partial y}{\partial x}\right) d x d t .
\end{aligned}
$$

It follows from (29) that

$$
A(y, v)=\int_{Q_{\tau}} x v(x, t) G\left(x, t, y, \frac{\partial y}{\partial x}\right) d x d t
$$

where

$$
A(y, v)=\int_{Q} x \frac{\partial y}{\partial t} v d x d t+a \int_{Q} x \frac{\partial y}{\partial x} \frac{\partial v}{\partial x} d x d t+b \int_{Q} x v y d x d t
$$

\section{Definition 4.1.}

A function $y \in L^{2}\left(0, T ; H_{\sqrt{x}}^{1}(0,1)\right)$ is said to be a weak solution of the problem $(25)-(27)$ if $(27)$ and (30) are fulfilled.

Constructing a recurrent sequence starting with $y^{(0)}=0$, and the sequence $\left(y^{(n)}\right)_{n \in \mathbb{N}}$ is defined as follows : given the element $y^{(n-1)}$, then for $n=1,2,3, \ldots$

We will solve the following problem :

$$
\left\{\begin{array}{c}
\frac{\partial y^{(n)}}{\partial t}-a \frac{1}{x} \frac{\partial}{\partial x}\left(x \frac{\partial y^{(n)}}{\partial x}\right)+b y^{(n)}=G\left(x, t, y^{(n-1)}, \frac{\partial y^{(n-1)}}{\partial x}\right) \\
y^{(n)}(x, 0)=0 \\
\frac{\partial y^{(n)}}{\partial x}(0, t)=\frac{\partial y^{(n)}}{\partial x}(1, t)=0
\end{array}\right.
$$

according to the theorem (3.2), each time we fix the $n$, the problem $(31))$ admits a unique solution $y^{(n)}(x, t)$. Suppose now $z^{(n)}(x, t)=y^{(n+1)}(x, t)-y^{(n)}(x, t)$, so we get the following new problem :

$$
\left\{\begin{array}{c}
\frac{\partial z^{(n)}}{\partial t}-a \frac{1}{x} \frac{\partial}{\partial x}\left(x \frac{\partial z^{(n)}}{\partial x}\right)=p^{(n-1)}(x, t) \\
z^{(n)}(x .0)=0 \\
\frac{\partial z^{(n)}}{\partial x}(0, t)=\frac{\partial z^{(n)}}{\partial x}(1, t)=0
\end{array}\right.
$$

where

$$
p^{(n-1)}(x, t)=G\left(x, t, y^{(n)}, \frac{\partial y^{(n)}}{\partial x}\right)-G\left(x, t, y^{(n-1)}, \frac{\partial y^{(n-1)}}{\partial x}\right)
$$


Lemma 4.1.

Suppose the condition (28) is satisfied. So for the problem (32)), we have a priori estimate:

$$
\left\|z^{n}\right\|_{L^{2}\left(0, T ; H_{\sqrt{x}}^{1}(0,1)\right)} \leq c\left\|z^{n-1}\right\|_{L^{2}\left(0, T ; H_{\sqrt{x}}^{1}(0,1)\right)}
$$

where

$$
c=\sqrt{\frac{k^{2} \exp \left(\frac{T}{2}\right)}{\min \left\{\frac{1}{2}, a, b\right\}}}
$$

Proof. By multiplying

$$
\frac{\partial z^{(n)}}{\partial t}-a \frac{1}{x} \frac{\partial}{\partial x}\left(x \frac{\partial z^{(n)}}{\partial x}\right)+b z^{(n)}=p^{(n-1)}(x, t),
$$

by $x z^{(n)}(x, t)$, and we integrate it on $Q_{\tau}$, we obtain :

$$
\begin{aligned}
& \int_{Q_{\tau}} \frac{\partial z^{(n)}}{\partial t}(x, t) \cdot x z^{(n)}(x, t) d x d t-a \int_{Q_{\tau}} \frac{\partial}{\partial x}\left(x \frac{\partial z^{(n)}}{\partial x}(x, t)\right) \cdot z^{(n)}(x, t) d x d t+b \int_{Q_{\tau}} x\left(z^{(n)}(x, t)\right)^{2} d x d t \\
= & \int_{Q_{\tau}} p^{(n-1)}(x, t) \cdot x z^{(n)}(x, t) d x d t .
\end{aligned}
$$

We use an integration by parts by taking into account the initial condition and the boundary condition, we find :

$$
\begin{aligned}
& \frac{1}{2} \int_{0}^{1} x\left(z^{(n)}(x, \tau)\right)^{2} d x+a \int_{Q_{\tau}} x\left(\frac{\partial z^{(n)}}{\partial x}(x, t)\right)^{2} d t+\int_{Q_{\tau}} b x\left(z^{(n)}(x, t)\right)^{2} d x d t . \\
\leq & \frac{1}{2} \int_{Q} x\left(z^{(n)}\right)^{2}+\frac{1}{2} \int_{Q} x\left(p^{n-1}\right)^{2}
\end{aligned}
$$

as $G$ is lipshitzian, we get :

$$
\begin{aligned}
& \frac{1}{2}\left\|z^{n}(x, \tau)\right\|_{L_{\sqrt{x}}^{2}(0,1)}^{2}+a\left\|\partial_{x} z^{n}\right\|_{L^{2}\left(0, T ; L_{\sqrt{x}}^{2}(0,1)\right)}^{2}+b\left\|z^{n}\right\|_{L^{2}\left(0, T ; L_{\sqrt{x}}^{2}(0,1)\right)}^{2} \\
\leq & \frac{1}{2}\left\|z^{n}\right\|_{L^{2}\left(0, T ; L_{\sqrt{x}}^{2}(0,1)\right)}^{2}+k^{2}\left\|z^{n-1}\right\|_{L^{2}\left(0, T ; H_{\sqrt{x}}^{1}(0,1)\right)}^{2},
\end{aligned}
$$

we apply Gronwall's lemma then pass to the maximum on $(0, T)$,we find

$$
\begin{aligned}
& \left\|z^{n}\right\|_{L^{\infty}\left(0, T ; L_{\sqrt{x}}^{2}(0,1)\right)}^{2}+\left\|z^{n}\right\|_{L^{2}\left(0, T ; H_{\sqrt{x}}^{1}(0,1)\right)}^{2} \\
\leq & \frac{k^{2} \exp \left(\frac{T}{2}\right)}{\min \left\{\frac{1}{2}, a, b\right\}}\left\|z^{n-1}\right\|_{L^{2}\left(0, T ; H_{\sqrt{x}}^{1}(0,1)\right)}^{2},
\end{aligned}
$$

So, we get

$$
\left\|z^{n}\right\|_{L^{2}\left(0, T ; H_{\sqrt{x}}^{1}(0,1)\right)} \leq c\left\|z^{n-1}\right\|_{L^{2}\left(0, T ; H_{\sqrt{x}}^{1}(0,1)\right)},
$$


where

$$
c=\sqrt{\frac{k^{2} \exp \left(\frac{T}{2}\right)}{\min \left\{\frac{1}{2}, a, b\right\}}},
$$

this achieved the demonstration.

According to the criterion of the convergence of the series, then $\sum_{n=1}^{\infty} z^{(n)}$ is said to be convergent if

$$
\sqrt{\frac{k^{2} \exp \left(\frac{T}{2}\right)}{\min \left\{\frac{1}{2}, a, b\right\}}}<1,
$$

Which implies that

$$
k<\sqrt{\frac{\min \left\{\frac{1}{2}, a, b\right\}}{\exp \left(\frac{T}{2}\right)}} .
$$

As $z^{(n)}(x, t)=y^{(n+1)}(x, t)-y^{(n)}(x, t)$, and $y^{(0)}(x, t)=0$, we have

$$
\sum_{i=0}^{n-1} z^{(i)}=y^{(n)}
$$

is convergente to an element $y \in L^{2}\left(0, T ; H_{\sqrt{x}}^{1}(0,1)\right)$.

Now,we will prove that $\lim _{n \longrightarrow \infty} y^{(n)}(x, t)=y(x, t)$ is a solution of the problem (31)) by showing that $y$ satisfies

$$
A(y, v)=\int_{Q_{\tau}} x v(x, t) G(x, t) d x d t
$$

Therefore we consider the weak formulation of the problem (31)) :

$$
A\left(y^{(n)}, v\right)=\int_{Q} x \frac{\partial y^{(n)}}{\partial t} v d x d t+a \int_{Q} x \frac{\partial y^{(n)}}{\partial x} \frac{\partial v}{\partial x} d x d t+b \int_{Q} x v y^{(n)} d x d t
$$

From the linearity of $A$ we have :

$$
\begin{aligned}
A\left(y^{(n)}, v\right)= & A\left(y^{(n)}-y, v\right)+A(y, v) \\
= & \int_{Q} x \frac{\partial\left(y^{(n)}-y\right)}{\partial t} v d x d t+a \int_{Q} x \frac{\partial\left(y^{(n)}-y\right)}{\partial x} \frac{\partial v}{\partial x} d x d t+b \int_{Q} x v\left(y^{(n)}-y\right) d x d t \\
& +A(y, v)
\end{aligned}
$$

we apply the Cauchy Schwartz inequality on $A\left(y^{(n)}-y, v\right)$, we get

$$
\begin{aligned}
& \int_{Q} x \frac{\partial\left(y^{(n)}-y\right)}{\partial t} v d x d t+a \int_{Q} x \frac{\partial\left(y^{(n)}-y\right)}{\partial x} \frac{\partial v}{\partial x} d x d t+b \int_{Q} x v\left(y^{(n)}-y\right) d x d t \\
\leq & 2 \max \{1, a, b\} \cdot\left\|v_{x}\right\|_{L^{2}\left(0, T ; H_{\sqrt{x}}^{1}(0,1)\right)}\left[\left\|\left(y^{(n)}-y\right)_{t}\right\|_{L^{2}\left(0, T ; H_{\sqrt{x}}^{1}(0,1)\right)}+\left\|\left(y^{(n)}-y\right)\right\|_{L^{2}\left(0, T ; H_{\sqrt{x}}^{1}(0,1)\right)}\right]
\end{aligned}
$$


On the other hand as

$$
y^{(n)} \longrightarrow y \quad \text { in } L^{2}\left(0, T ; H_{\sqrt{x}}^{1}(0,1)\right)
$$

so

$$
\begin{aligned}
& y^{(n)} \longrightarrow y \quad \text { in } L^{2}\left(0, T ; L_{\sqrt{x}}^{2}(0,1)\right) \\
& y_{t}^{(n)} \longrightarrow y_{t} \quad \text { in } L^{2}\left(0, T ; L_{\sqrt{x}}^{2}(0,1)\right) \\
& y_{x}^{(n)} \longrightarrow y_{x} \quad \text { in } L^{2}\left(0, T ; L_{\sqrt{x}}^{2}(0,1)\right)
\end{aligned}
$$

Let us pass to the limit when $n \longrightarrow+\infty$, we find

$$
\lim _{n \longrightarrow+\infty} A\left(y^{(n)}-y, v\right)=0 .
$$

According to (35) and by passing to the limit in (34) we obtain

$$
\lim _{n \longrightarrow+\infty} A\left(y^{(n)}, v\right)=A(y, v)
$$

Thus, we have proved the following result :

\section{Theorem 4.1.}

If the condition (28) is satisfied. And

$$
k<\sqrt{\frac{\min \left\{\frac{1}{2}, a, b\right\}}{\exp \left(\frac{T}{2}\right)}} .
$$

Then the problem (25) admits a weak solution belonging to $L^{2}\left(0, T ; H_{\sqrt{x}}^{1}(0,1)\right)$.

Now,we will show that the solution of the problem $(25)-(27)$ is unique.

\section{Theorem 4.2.}

If the condition (28) is verified, then the solution is unique.

Proof. Let $y_{1}, y_{2}$ in $L^{2}\left(0, T ; H^{1}(0,1)\right)$ be two solution of $(25)-(27)$, then

$$
y=y_{1}-y_{2},
$$

is also a solution in $L^{2}\left(0, T ; H_{\sqrt{x}}^{1}(0,1)\right)$ and verifies

$$
\frac{\partial y}{\partial t}-a \frac{1}{x} \frac{\partial}{\partial x}\left(x \frac{\partial y}{\partial x}\right)+b y=G\left(x, t, y, \frac{\partial y}{\partial x}\right)
$$




$$
\begin{aligned}
y(x, 0) & =0, \\
\frac{\partial y}{\partial x}(0, t) & =\frac{\partial y}{\partial x}(1, t)=0,
\end{aligned}
$$

so, we get

$$
\begin{aligned}
\frac{\partial y}{\partial t}-a \frac{1}{x} \frac{\partial}{\partial x}\left(x \frac{\partial y}{\partial x}\right)+b y & =\Psi(x, t), \quad \forall(x, t) \in Q \\
y(x, 0) & =0 \\
\frac{\partial y}{\partial x}(0, t) & =\frac{\partial y}{\partial x}(1, t)=0
\end{aligned}
$$

where

$$
\Psi(x, t)=G\left(x, t, y_{1}, \frac{\partial y_{1}}{\partial x}\right)-G\left(x, t, y_{2}, \frac{\partial y_{2}}{\partial x}\right) .
$$

Using the lemma (4.1) we get

$$
\|y\|_{L^{2}\left(0, T, H_{\sqrt{x}}^{1}(0,1)\right)} \leq c\|y\|_{L^{2}\left(0, T, H_{\sqrt{x}}^{1}(0,1)\right)}
$$

from where

$$
(1-c)\|y\|_{L^{2}\left(0, T, H_{\sqrt{x}}^{1}(0,1)\right)} \leq 0
$$

and as $c \leq 1$, we get then

$$
\|y\|_{L^{2}\left(0, T, H_{\sqrt[x]{x}}^{1}(0,1)\right)}=0
$$

from where

$$
y_{1}=y_{2},
$$

which gives the uniqueness of the solution.

\section{Formulation of the nonlinear problem}

Let $Q=\left\{(x, t) \in \mathbb{R}^{2}, 0<x<1\right.$ and $\left.0<t<T\right\}$.

Consider the following nonlinear problem :

$$
\begin{cases}\frac{\partial u}{\partial t}-a \frac{1}{x} \frac{\partial}{\partial x}\left(x \frac{\partial u}{\partial x}\right)+b u=u^{p}, & \forall(x, t) \in Q \\ u(x, 0)=\varphi(x), & \forall x \in(0,1) \\ \frac{\partial u}{\partial x}(0, t)=\frac{\partial u}{\partial x}(1, t)=0 . & \forall t \in(0, T)\end{cases}
$$

Such that $a, b>0, f, \varphi \in L^{2}(Q)$ are known functions, and by integrating on the $\Omega=(0,1)$, we obtain :

$$
\int_{\Omega}\left[u_{t}(x, t)-a \frac{1}{x} \partial_{x}\left(x \partial_{x} u(x, t)\right)+b u(x, t)\right] \cdot x u(x, t) d x=\int_{\Omega} x u^{p+1}(x, t) d x .
$$


Therefore

$$
\begin{aligned}
& \int_{\Omega} u_{t}(x, t) \cdot x u(x, t) d x-a \int_{\Omega} \partial_{x}\left(x \partial_{x} u(x, t)\right) \cdot u(x, t) d x \\
& \quad+b \int_{\Omega} u(x, t) \cdot x u(x, t) \\
& =\int_{\Omega} x u^{p+1}(x, t) d x d t .
\end{aligned}
$$

Then, by using the integration by part and the Neumann conditions, we get :

$$
\frac{1}{2} \frac{d}{d t} \int_{\Omega} x u^{2}(x, t) d x+a \int_{\Omega} x\left(\partial_{x} u(x, t)\right)^{2} d x+b \int_{\Omega} x u^{2}(x, t) d x=\int_{\Omega} x u^{p+1}(x, t) d x .
$$

So, it comes

$$
\begin{gathered}
\frac{1}{2} \frac{d}{d t} \int_{\Omega} x u^{2}(x, t) d x-\frac{p-1}{p+1} \int_{\Omega} x u^{p+1}(x, t) d x \\
+\left[a \int_{\Omega} x\left(\partial_{x} u(x, t)\right)^{2} d x+b \int_{\Omega} x u^{2}(x, t) d x-\frac{2}{p+1} \int_{\Omega} x u^{p+1}(x, t) d x\right]=0
\end{gathered}
$$

By putting

$$
E(t)=a \int_{\Omega} x\left(\partial_{x} u(x, t)\right)^{2} d x+b \int_{\Omega} x u^{2}(x, t) d x-\frac{2}{p+1} \int_{\Omega} x u^{p+1}(x, t) d x
$$

Where $E$ called the energy function, we find :

$$
\frac{1}{2} \frac{d}{d t} \int_{\Omega} x u^{2}(x, t) d x+E(t)-\frac{p-1}{p+1} \int_{\Omega} x u^{p+1}(x, t) d x=0
$$

Then multiplying the main equation by $u_{t}$ and integrating over $\Omega$ yields

$$
\begin{aligned}
& \int_{\Omega} x u_{t}^{2}(x, t) d x+\frac{a}{2} \frac{d}{d t} \int_{\Omega} x\left(\partial_{x} u(x, t)\right)^{2} d x+\frac{b}{2} \frac{d}{d t} \int_{\Omega} x u^{2}(x, t) d x=\frac{1}{p+1} \frac{d}{d t} \int_{\Omega} x u^{p+1}(x, t) d x \\
& \int_{\Omega} x u_{t}^{2}(x, t) d x+\frac{1}{2} \frac{d}{d t}\left[a \int_{\Omega} x\left(\partial_{x} u(x, t)\right)^{2} d x+b \int_{\Omega} x u^{2}(x, t) d x-\frac{2}{p+1} \int_{\Omega} x u^{p+1}(x, t) d x\right]=0 \\
& \int_{\Omega} x u_{t}^{2}(x, t) d x+\frac{1}{2} \frac{d E(t)}{d t}=0
\end{aligned}
$$


This implies that $E$ decresing and if $E(0)<0$, then and by appling Jensen inequality

$$
\begin{aligned}
& \frac{1}{2} \frac{d}{d t} \int_{\Omega} x u^{2}(x, t) d x>\frac{p-1}{p+1} \int_{\Omega} x u^{p+1}(x, t) d x \\
& \frac{1}{2} \frac{d}{d t} \int_{\Omega} x u^{2}(x, t) d x>\frac{(p-1)}{p+1}\left(\frac{1}{2}\right)^{\frac{p-1}{2}}\left(\int_{\Omega} x u^{2}(x, t) d x\right)^{\frac{P+1}{2}}
\end{aligned}
$$

By putting

$$
\Pi(t)=\int_{\Omega} x u^{2}(x, t) d x
$$

Then, we get

$$
\begin{aligned}
\frac{d \Pi(t)}{d t} & >\frac{2(p-1)\left(\frac{1}{2}\right)^{\frac{p-1}{2}}}{p+1}(\Pi(t))^{\frac{P+1}{2}} \\
\frac{d \Pi(t)}{(\Pi(t))^{\frac{P+1}{2}}} & >\frac{2(p-1)\left(\frac{1}{2}\right)^{\frac{p-1}{2}}}{p+1} d t
\end{aligned}
$$

Therefore, after integrating over $0, t$

$$
\begin{aligned}
& -\frac{2}{1-p}\left[\frac{1}{(\Pi(t))^{\frac{P-1}{2}}}-\frac{1}{(\Pi(0))^{\frac{P-1}{2}}}\right]>\frac{2(p-1)\left(\frac{1}{2}\right)^{\frac{p-1}{2}}}{p+1} t \\
& \frac{2}{1-p}\left[\frac{1}{(\Pi(t))^{\frac{P-1}{2}}}-\frac{1}{(\Pi(0))^{\frac{P-1}{2}}}\right]<\frac{2(p-1)\left(\frac{1}{2}\right)^{\frac{p-1}{2}}}{p+1} t \\
& \frac{1}{(\Pi(t))^{\frac{P-1}{2}}}<\frac{-(p-1)^{2}\left(\frac{1}{2}\right)^{\frac{p-1}{2}}}{p+1} t+\frac{1}{(\Pi(0))^{\frac{P-1}{2}}} \\
& \left(\frac{1}{\frac{-(p-1)^{2}\left(\frac{1}{2}\right)^{\frac{p-1}{2}}}{p+1} t+\frac{1}{(\Pi(0))^{\frac{P-1}{2}}}}\right)^{\frac{P-1}{2}}<\Pi(t)
\end{aligned}
$$

This implies that when $t \longrightarrow T^{*}$ with

$$
T^{*}=\frac{\frac{1}{(\Pi(0))^{\frac{P-1}{2}}}}{\frac{(p-1)^{2}\left(\frac{1}{2}\right)^{\frac{p-1}{2}}}{p+1}}
$$

the solution $u$ must blow up.

\section{References}

[1] Bouziani Abdelfatah, Oussaeif Taki-Eddine, and Ben Aoua Leila,A Mixed Problem with an Integral TwoSpace-Variables Condition for Parabolic Equation with The Bessel Operator, Journal of Mathematics. 2013 (2013), Article ID 457631. 
[2] Dhelis Sofiane, Bouziani Abdelfatah, Oussaeif Taki-Eddine. Study of Solution for a Parabolic Integrodifferential Equation with the Second Kind Integral Condition, Int. J. Anal. Appl., 6 (4) (2018), 569-593.

[3] O. Taki-Eddine, B Abdelfatah; A priori estimates for weak solution for a time-fractional nonlinear reactiondiffusion equations with an integral condition,Chaos, Solitons Fractals, 103 (2017), 79-89.

[4] Taki-Eddine Oussaeif, Bouziani Abdelfatah; Inverse problem of a hyperbolic equation with an integral overdetermination condition, Electronic Journal of Differential Equations, 2016(138) (2016), 1-7.

[5] T-E Oussaeif, A Bouziani; Existence and uniqueness of solutions to parabolic fractional differential equations with integral conditions, Electronic Journal of Differential Equations; 2014(179) (2014), 1-10.

[6] T-E Oussaeif, A Bouziani, Mixed Problem with an Integral Two-SpaceVariables Condition for a Parabolic Equation. International Journal of Evolution Equations, 9(9), 181-198.

[7] Taki-Eddine Oussaeif and Abdelfatah Bouziani. Mixed Problem with an Integral Two-Space-Variables Condition for a Class of Hyperbolic Equations. International Journal of Analysis, 2013 (2013), Article ID 957163, 8 pages.

[8] T.-E. Oussaeif, A. Bouziani.Solvability of nonlinear viscosity equation with a boundary integral condition. J. Nonl. Evol. Equ. Appl. 3 (2015), 31-45.

[9] Oussaeif Taki Eddine,Bouziani Abdelfatah.Mixed Problem with an Integral Two-Space-Variables condition for a Third Order Parabolic Equation. International Journal of Analysis and Applications, 12(2) (2016), 98-117.

[10] Oussaeif Taki-Eddine, Bouziani Abdelfatah. Nonlocal problem for a third order partial differential equation of mixed type with an integral two-spacevariables condition, Communications in Optimization Theory. 2017 (2017), 1-16, Article ID 16.

[11] Oussaeif Taki-Eddine, Bouziani Abdelfatah. On a class of hyperbolic equation with an integral two-spacevariables condition with the bessel operator; Advanced Studies in Contemporary Mathematics. 28(1) (2018), $83-92$.

[12] Oussaeif Taki-Eddine, Bouziani Abdelfatah. Solvability of Nonlinear Goursat Type Problem for Hyperbolic Equation with Integral Condition; Khayyam Journal of Mathematics 4, 2018 (2018), 94-102. 